\title{
The mechanism of Bushen Huoxue decoction in treating intervertebral disc degeneration based on network pharmacology
}

\author{
Shuai-Hua Feng ${ }^{1 \#}$, Fang Xie ${ }^{2 \#}$, Hong-Yan Yao $^{3}$, Guan-Bao Wu ${ }^{1}$, Xiang-Yun Sun ${ }^{1}$, Jie Yang ${ }^{1}$ \\ ${ }^{1}$ Department of Bone Traumatology, Affiliated Hospital of Hunan Academy of Chinese Medicine, Changsha, China; ${ }^{2}$ Graduate School of Education, \\ Hunan University of Traditional Chinese Medicine, Changsha, China; ${ }^{3}$ School Hospital, Hunan University of Traditional Chinese Medicine, \\ Changsha, China \\ Contributions: (I) Conception and design: SH Feng, F Xie, GB Wu; (II) Administrative support: GB Wu; (III) Provision of study materials or patients: \\ SH Feng, F Xie, GB Wu; (IV) Collection and assembly of data: HY Yao; (V) Data analysis and interpretation: XY Sun, J Yang; (VI) Manuscript \\ writing: All authors; (VII) Final approval of manuscript: All authors. \\ \#These authors contributed equally to this work. \\ Correspondence to: Guan-Bao Wu. Department of Bone Traumatology, Affiliated Hospital of Hunan Academy of Chinese Medicine, No. 58 of Lushan \\ road, Yuelu District, Changsha 410006, China. Email: gbwdr_sun09@163.com.
}

\begin{abstract}
Background: This study aimed to explore the mechanism of Bushen Huoxue decoction (BHD) in treating intervertebral disc degeneration using the network pharmacology method.

Methods: Using of oral bioavailability $>30 \%$ and drug-likeness $>0.18$ as the screening standards, the effective components and targets of BHD were retrieved from the TCMSP database and the BATMANTCM database. The disease targets of intervertebral disc degeneration were retrieved from the GeneCards database. The Wayne map of the interaction targets of the effective components of BHD and intervertebral disc degeneration were drawn using $\mathrm{R}$ software. The protein-protein interaction (PPI) network of common targets was constructed using STRING software. The network map of the interaction targets of the effective components of BHD-intervertebral disc degeneration was drawn using Cytoscape3.7.2 software. The GO and KEGG enrichment analysis of the common targets of BHD and intervertebral disc degeneration was performed using $\mathrm{R}$ software and the related plug-ins to screen the potential pathways and analyze its mechanism.
\end{abstract}

Results: This study screened 164 effective components of BHD, 131 interaction targets, 626 targets for degenerative disc disease, and 31 common interaction targets. IL6, VEGFA, CASP3, EGFR, ESR1, and MAPK8 appeared more frequently. These were mainly enriched in the AGE-RAGE, TNF, PI3K Akt, and MAPK signaling pathways.

Conclusions: BHD mainly intervenes in intervertebral disc degeneration through IL6, VEGFA, CASP3, EGFR, ESR1, and MAPK8. The mechanism of the intervention of BHD on intervertebral disc degeneration may be related to AGE-RAGE, TNF, PI3K Akt, MAPK, and other signal pathways.

Keywords: Network pharmacology; tonifying kidney and activating blood soup; intervertebral disc degeneration; the network diagram; common target protein interaction network

Submitted Jul 17, 2020. Accepted for publication Jan 02, 2021.

doi: 10.21037/apm-20-2586

View this article at: http://dx.doi.org/10.21037/apm-20-2586

\section{Introduction}

Intervertebral disc degeneration (IDD) is a chronic progressive disease caused by many factors. Its pathological changes are characterized by the decrease in proliferation activity, the quantity of nucleus pulposus cells, and the decrease in extracellular matrix synthesis $(1,2)$. IDD is one of the main causes of chronic low back pain and the basis of various spinal diseases such as intervertebral disc herniation and 


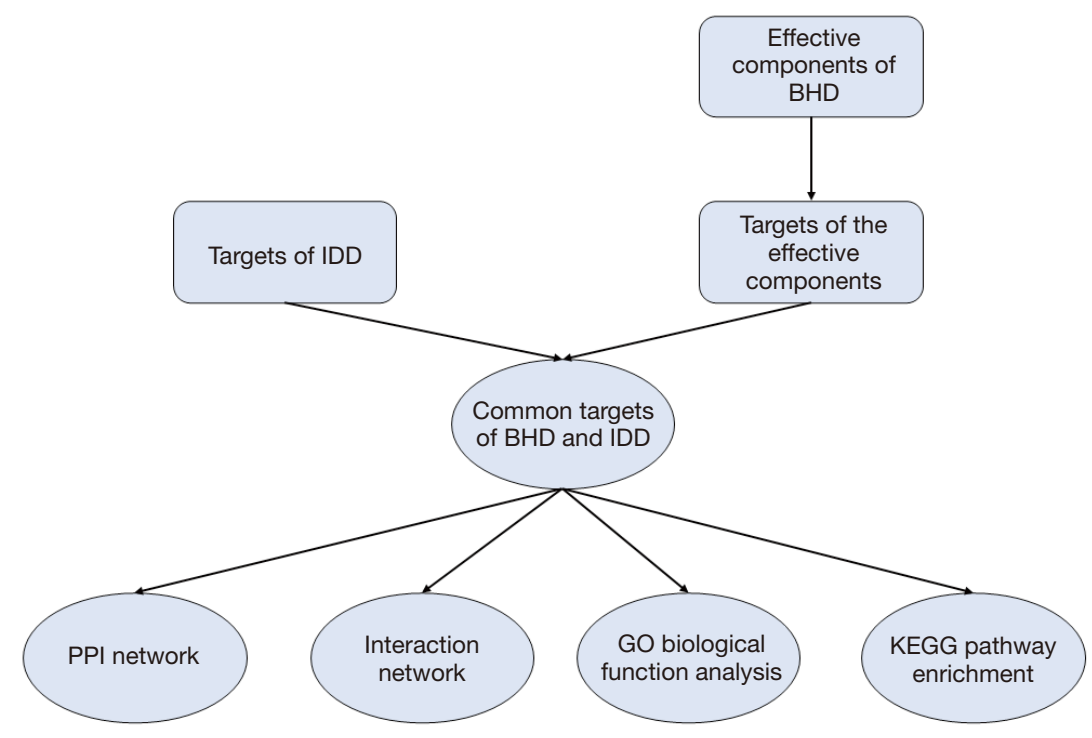

Figure 1 The study flow chart. BHD, bushen huoxue decoction; IDD, intervertebral disc degeneration; PPI, protein-protein interaction.

spondylolisthesis. It seriously affects patients' quality of life and labor ability and imposes a great burden on the social economy.

Traditional Chinese medicine believes that improving renal function can effectively delay low back pain caused by IDD. Bushen Huoxue decoction (BHD) was recorded in the Great Achievement of Traumatology, written by Zhao Lian, a physician in the Qing Dynasty. It can effectively improve renal function and promote blood circulation. It is a commonly used prescription in the treatment of degenerative diseases of intervertebral discs in traditional Chinese medicine, with excellent clinical effect (3). Previous studies revealed that BHD could inhibit the adipogenic differentiation of bone marrow mesenchymal stem cells (BMSCs), promote their osteogenic differentiation and migration in vitro, and regulate the expression of IL- $1 \beta$ (4), TGF- $\beta 1(5,6)$, and p38MAPK proteins (7) in degenerative discs. BHD can also regulate multiple targets and multiple mechanisms. Although we have uncovered part of the mechanism of BHD in the treatment of degenerative disc disease, this is just the tip of the iceberg. More complex regulatory mechanisms need to be explored and reviewed.

Previous studies on the regulation mechanism of BHD on IDD are generally one-sided, lacking macroscopic and overall understanding. Therefore, based on network pharmacology technology, this study explored the corresponding relationship between drug components and disease targets, predicted all possible regulatory mechanisms, and provided a theoretical basis for the follow-up experimental study of BHD in the treatment of IDD. The study flow chart is shown in Figure 1. We present the following article in accordance with the MDAR reporting checklist (available at http://dx.doi.org/10.21037/apm-20-2586).

\section{Methods}

The study was conducted in accordance with the Declaration of Helsinki (as revised in 2013). The study was approved by ethics board of Affiliated Hospital of Hunan Academy of Chinese Medicine (No. LLBH-201910310001) and informed consent was taken from all the patients.

\section{Effective components and the selection of targets of BHD}

The TCMSP database (http://lsp.nwu.edu.cn/tcmsp. php) and BATMAN-TCM database (http://bionet. ncpsb.org/batman-tcm/) were used to retrieve all the effective components of the raw materials of BHD: Radix Rehmanniae, Eucommia ulmoides Oliv, Fructus Lycii, Fructus Psoraleae, Dodder, Angelicae Sinensis Radix, myrrh, Fructus corni, safflower, Angelica, and Cistanche deserticola. The preliminarily screened literature was read according to the criteria of oral bioavailability (OB) $\geq 30 \%$ and drug-likeness (DL) $\geq 0.18$. The primarily screened literature was re-screened, thus, obtaining the effective components of BHD. Subsequently, through the TCMSP and BATMAN-TCM database, all the targets of each 
component of BHD were retrieved. The targets and the effective components of BHD were then inputted into the Perl plug-ins.

\section{The screening of targets for degenerative disc disease}

The disease targets of disc degeneration were searched through the GeneCards database (https://www.genecards. org/) using the search term "Intervertebral disc degeneration". The inclusion criterion was a relevance score of $\geq 17.0$. After eliminating the repeated targets, the disease targets of disc degeneration were standardized through the UniProt database (https://www.uniprot.org).

\section{The screening of common targets of active ingredients, disease, and PPI network construction}

To clarify the interaction between the related targets of IDD and the potential targets of BHD, the R language (https://www.r-project.org/) software and the Perl language program were used to intersect the disease targets and drug targets. After, the Wayne map was drawn. The STRING (https://string-db.org/) plug-in was used to build a common target protein-protein interaction (PPI) network. The protein type was set to "Homo sapiens", and other parameters remained as the default settings to obtain the PPI network. The Count. R plug-in was used to obtain the frequency of common protein targets.

\section{The construction of the interaction network of the effective component-disc degeneration-target}

The Merge function of the Cytoscape 3.7.1 software was used to construct the interaction network of the effective component-disc degeneration-target. In the network diagram, "Node" represented the effective components and targets, and "edge" represented the relationship between the effective component-BHD-target.

\section{Gene Ontology (GO) biological function analysis and the KEGG patbway enrichment}

In the Bioconductor database (http://www.bioconductor. org/) after the data download, R software was used (https:// www.r-project.org/). GO enrichment analysis and KEGG enrichment analysis were carried out automatically for drug and disease interaction targets, and Perl plug-in was used to transform and output the results. GO analysis is mainly used to describe the function of gene products, including cell function, molecular function, and biological function. KEGG analysis mainly describes the location and role of the target in related biological signal pathways.

\section{Statistical analysis}

All the GO and KEGG enrichment analysis results were selected by $\mathrm{P}<0.05$ as the critical criterion, and the first 20 pathways were screened out.

\section{Results}

\section{The screening of the active components of $B H D$}

In the present study, using the TCMSP and BATMANTCM database, $\mathrm{OB} \geq 30 \%$ and $\mathrm{DL} \geq 0.18$ were used as the inclusion criteria for screening to search the active components of 11 Chinese herbs in BHD. Then, 192 active components were obtained. Among these, Eucommia had 28 active components, radix angelicae tuhuo nine, wolfberry 45 , angelica 28 , safflower 22 , myrrh 45 , Cistanche deserticola six, Cornus 20, prepared Radix Rehmanniae two, Dodder 11, and Psoralea had two. After eliminating the repeated active components, 164 active components were obtained. A total of 10,442 targets of BHD were retrieved from the TCMSP and BATMAN-TCM database, and the Perl plug-in was used to screen the targets of the active ingredients of BHD. After eliminating the repeated parts, 131 targets were obtained.

\section{The screening targets for degenerative disc disease}

In the present study, the disease targets of IDD were retrieved from the GeneCards and OMIM database. The relevance score of $\geq 17.0$ was used as the inclusion criterion. After eliminating the repeated targets, 630 therapeutic targets of IDD were obtained.

\section{Common target screening and the construction of the PPI network}

The R language software and Venny plug-in program were used to screen the common targets from 661 targets of intervertebral disc degenerative disease and 131 active drug components. A Wayne map was drawn (Figure 2). Then, 31 common targets were input in the STRING database (https://string-db.org/), the human genome was chosen, and 
the PPI network was constructed in the mode of "medium confidence: 0.4" (Figure 3). The Count. R plug-in was used to count the top 30 protein targets. The findings showed that IL6, VEGFA, CASP3, EGFR, ESR1, and MAPK8 appeared more frequently (Figure 4).

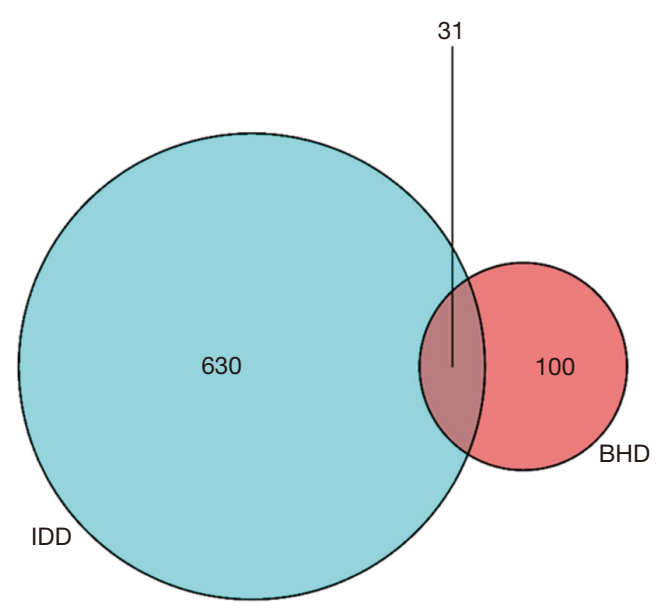

Figure 2 Venny diagram of components target of BHD and target of IDD. BHD, bushen huoxue decoction; IDD, intervertebral disc degeneration.

\section{The construction of the interaction network of the active components of BHD-intervertebral disc degeneration-target}

The Cytoscape 3.7.1 software was used to obtain 77 nodes and 384 edges from the interaction network diagram of

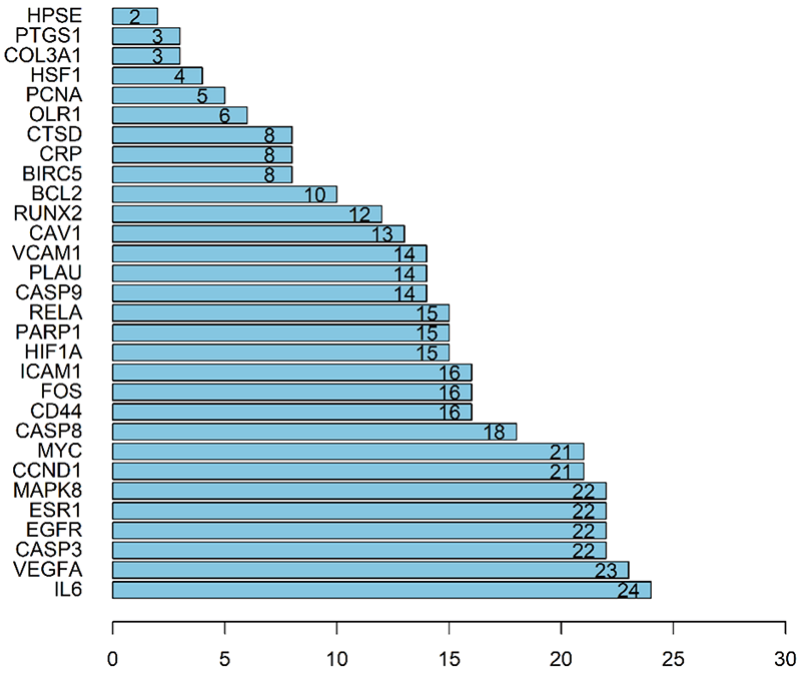

Figure 4 Frequency of common protein targets.

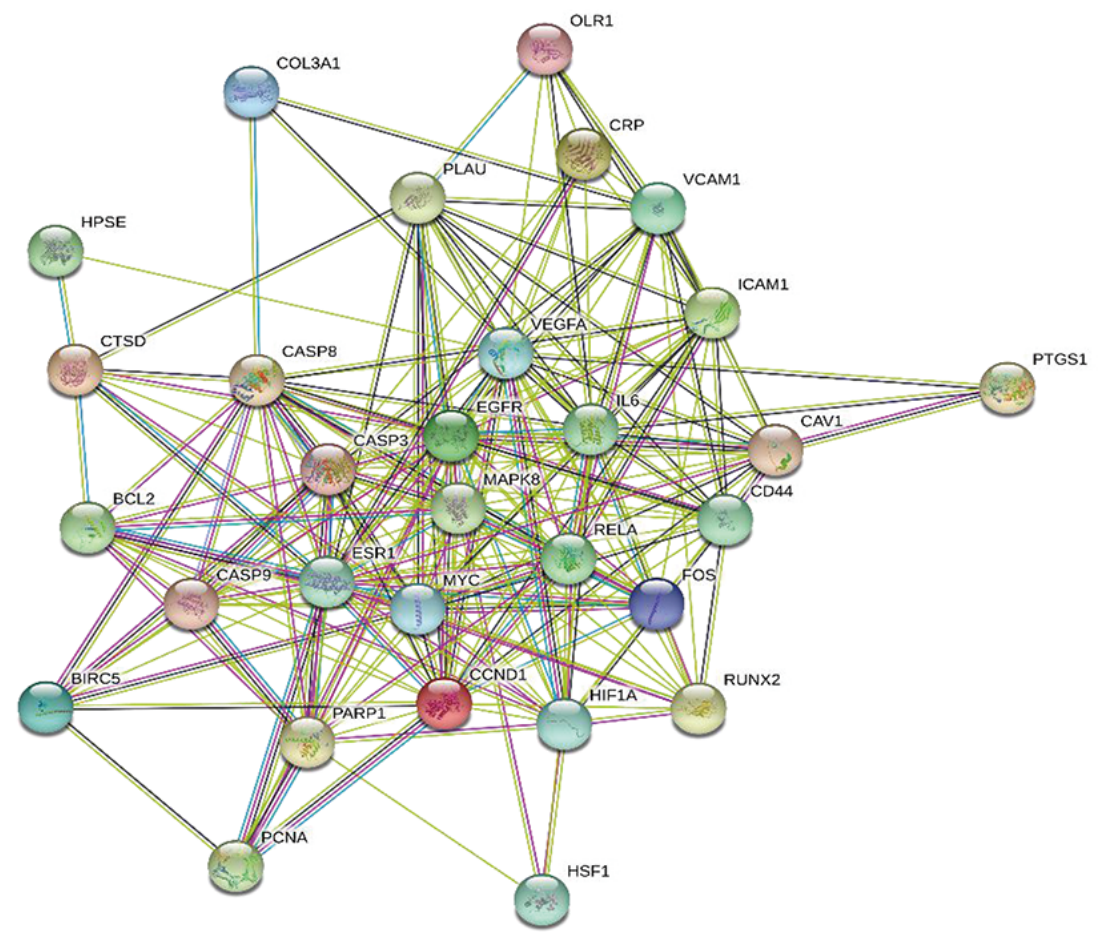

Figure 3 PPI network of BHD and IDD. BHD, bushen huoxue decoction; IDD, intervertebral disc degeneration. 


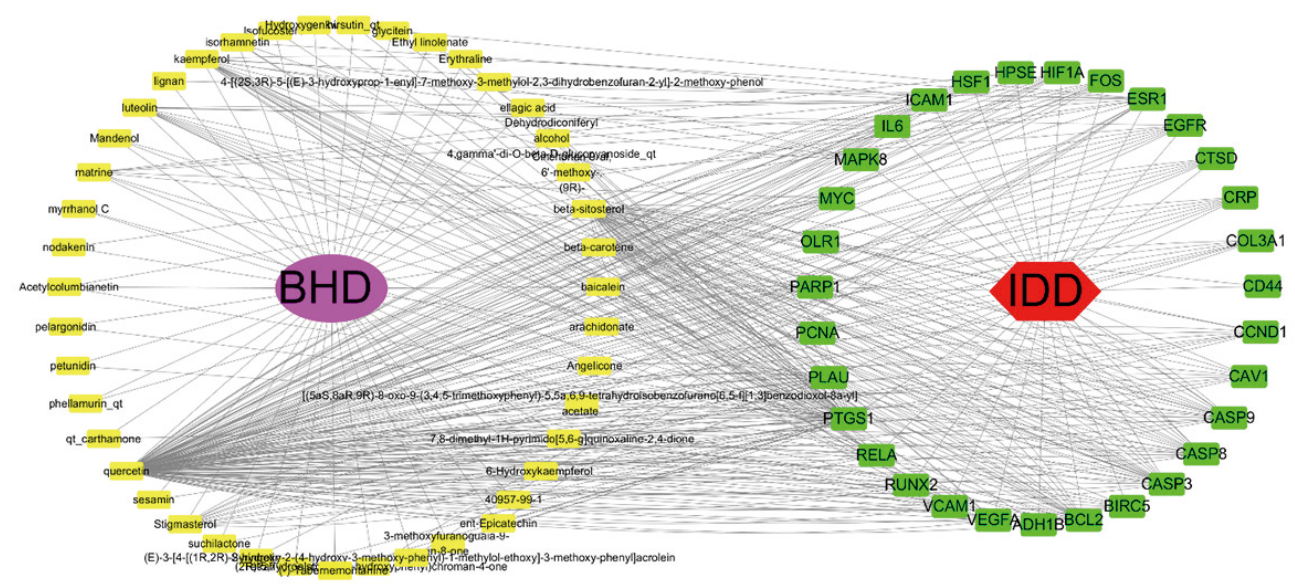

Figure 5 Active constituents-IDD-target interaction network. In the picture, purple is BHD, red is the disease disc degeneration, yellow is the active ingredient of BHD, and green is the common target of BHD and IDD. BHD, bushen huoxue decoction; IDD, intervertebral disc degeneration.

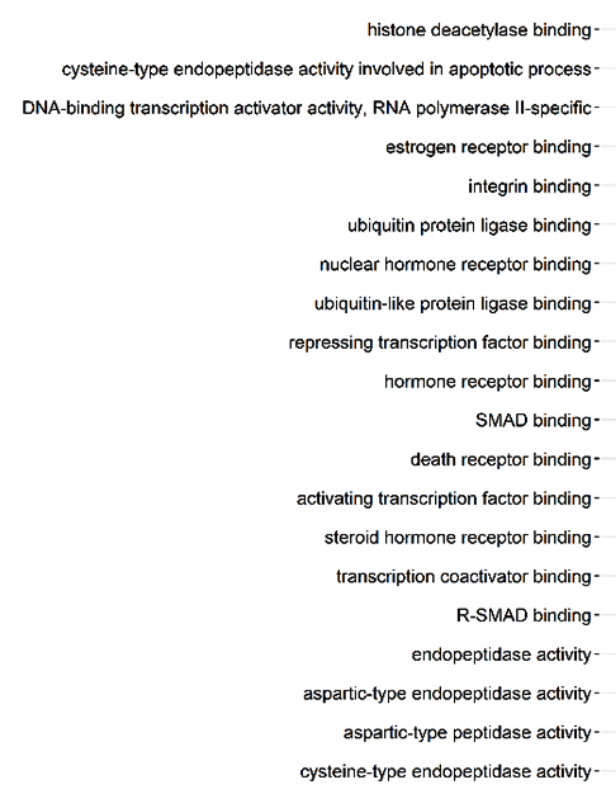

Figure 6 Histogram of GO analysis of common target.

active component-intervertebral disc degeneration-target (Figure 5). In Figure 5, purple is the drug BHD, red is the disease disc degeneration, yellow is the active component of $\mathrm{BHD}$, and green is the common targets of BHD and IDD.

\section{The enrichment analysis of BHD in the treatment of intervertebral disc degeneration}

The Gene Ontology (GO) enrichment analysis of the

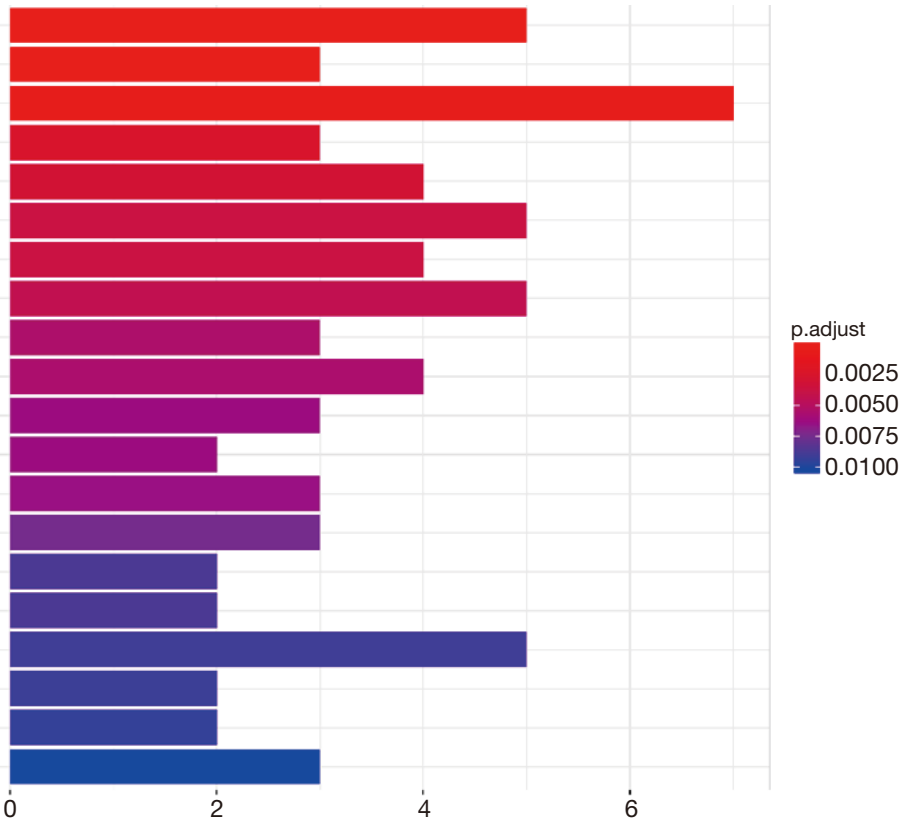

molecular function of the common targets of BHD and IDD was performed. Figures 6 and 7 show the top 20 molecular functions. DNA binding transcription activator activity, RNA polymerase II specific, ubiquitinprotein ligase binding, histone deacetylase binding, and ubiquitin-like protein ligase binding are ranked in the top five positions. The Kyoto Encyclopedia of Genes and Genomes (KEGG) biological pathway enrichment analysis of the common targets was also carried out. Figures 8 and 9 


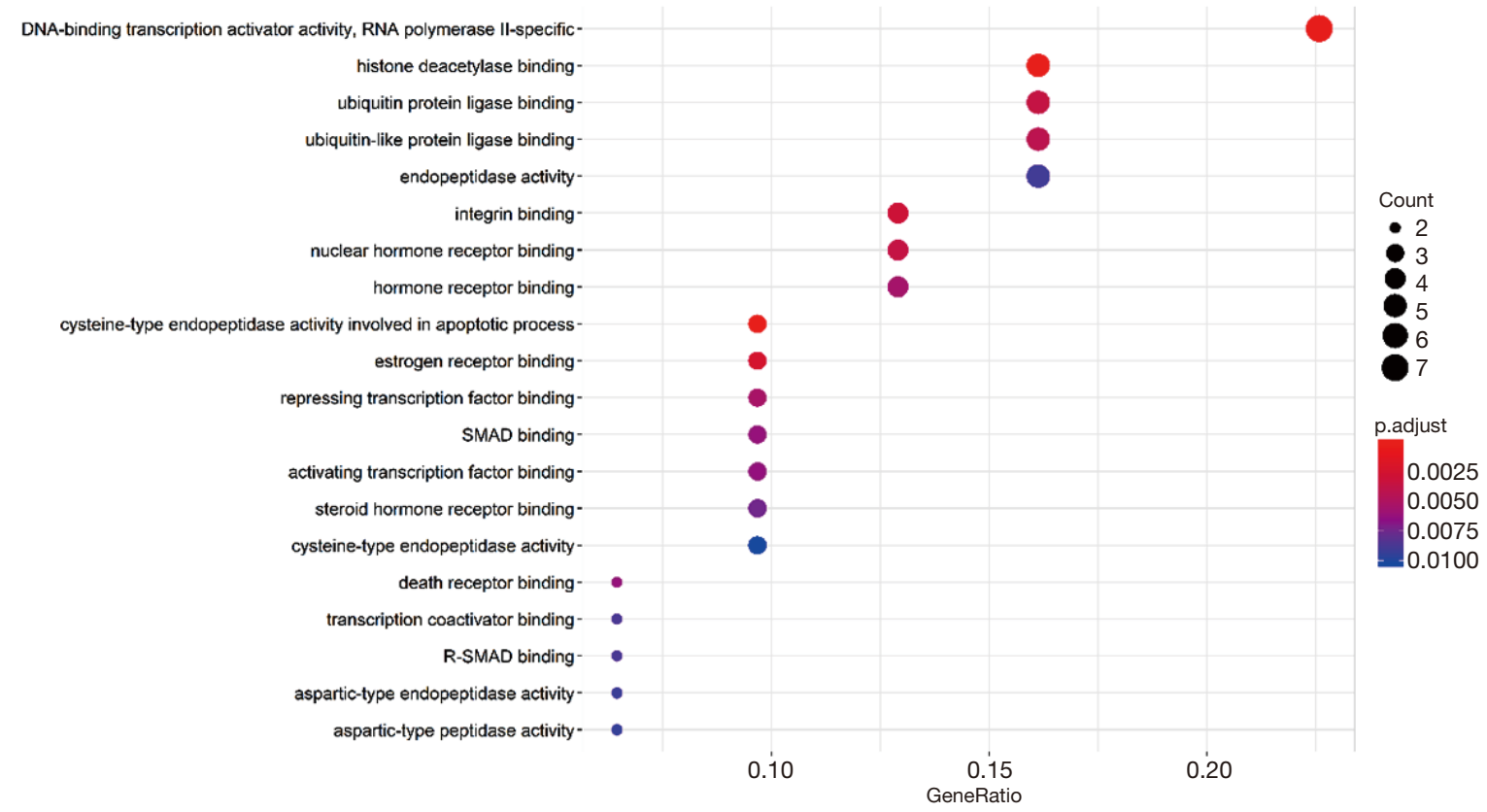

Figure 7 Bubble chart of GO analysis of common target.

show the top 20 biological pathways. The results revealed that the top four enrichment frequency of biological pathways of interaction targets of BHD on IDD were Kaposi sarcoma-associated herpesvirus infection (KSHV), hepatitis B, Epstein-Barr virus infection, and proteoglycans in cancer. The results of the present study revealed that the targets of BHD intervention on IDD are mainly enriched in the AGE-RAGE signaling pathway, TNF signaling pathway, PI3K Akt signaling pathway, MAPK signaling pathway, Relaxin signaling pathway, NF kappa B signaling pathway, HIF-1 signaling pathway, prolactin signaling pathway, $\mathrm{p} 53$ signaling pathway, toll-like receptors signaling pathway, gyroid hormone signaling pathway, Estrogen signaling pathway, JAK-STAT signaling pathway, and nodelike receptor signaling pathway.

\section{Discussion}

In the present study, 164 effective components and 131 targets of BHD and 626 targets of IDD were screened, and 31 common interaction targets were obtained. IL6, VEGFA, CASP3, EGFR, ESR1, and MAPK8 appeared more frequently, which were mainly enriched in the AGERAGE, TNF, PI3K Akt, and MAPK signaling pathways.

The specific mechanism of IDD remains unknown. The biological characteristics of its degeneration are tissue fibrosis caused by the progressive decrease of proteoglycan, dehydration of type II collagen, and an increase in type I collagen (8-10). During the process, interleukin-1 (IL-1), tumor necrosis factor $\alpha(\mathrm{TNF}-\alpha)$, and other proinflammatory factors affect the balance of the extracellular matrix by stimulating the production of NO, matrix metalloproteinase, and proteoglycans while resulting in the degradation of the extracellular matrix and the degeneration of the pulpiform nucleus and fibrous rings (11-13). At present, research in traditional Chinese medicine puts the intervertebral disc into the category of muscles and bones, which is closely related to the liver and kidneys. BHD is from "The Great Achievement of Traumatology", written by Lian Zhao in the Qing Dynasty. During the decoction intervention, prepared Rehmannia and Eucommia are considered as sovereign medicine. These tonify the liver and kidneys and strengthen the muscles and bones. Cornus Officinalis, wolfberry, Cistanche deserticola, Psoralea, and Cuscuta are ministerial drugs that warm Yang and tonify the kidneys. Angelicae Sinensis Radix, myrrh, and safflower are conducting drugs that clear the channels and remove blood stasis. Angelica eliminates wind and dampness, disperses arthralgia, and relieves pain. The decoction tonifies the liver and kidneys, removes rheumatism, strengthens the body's resistance, eliminates pathogens, clears channels, and removes blood stasis. Drugs are used according to 


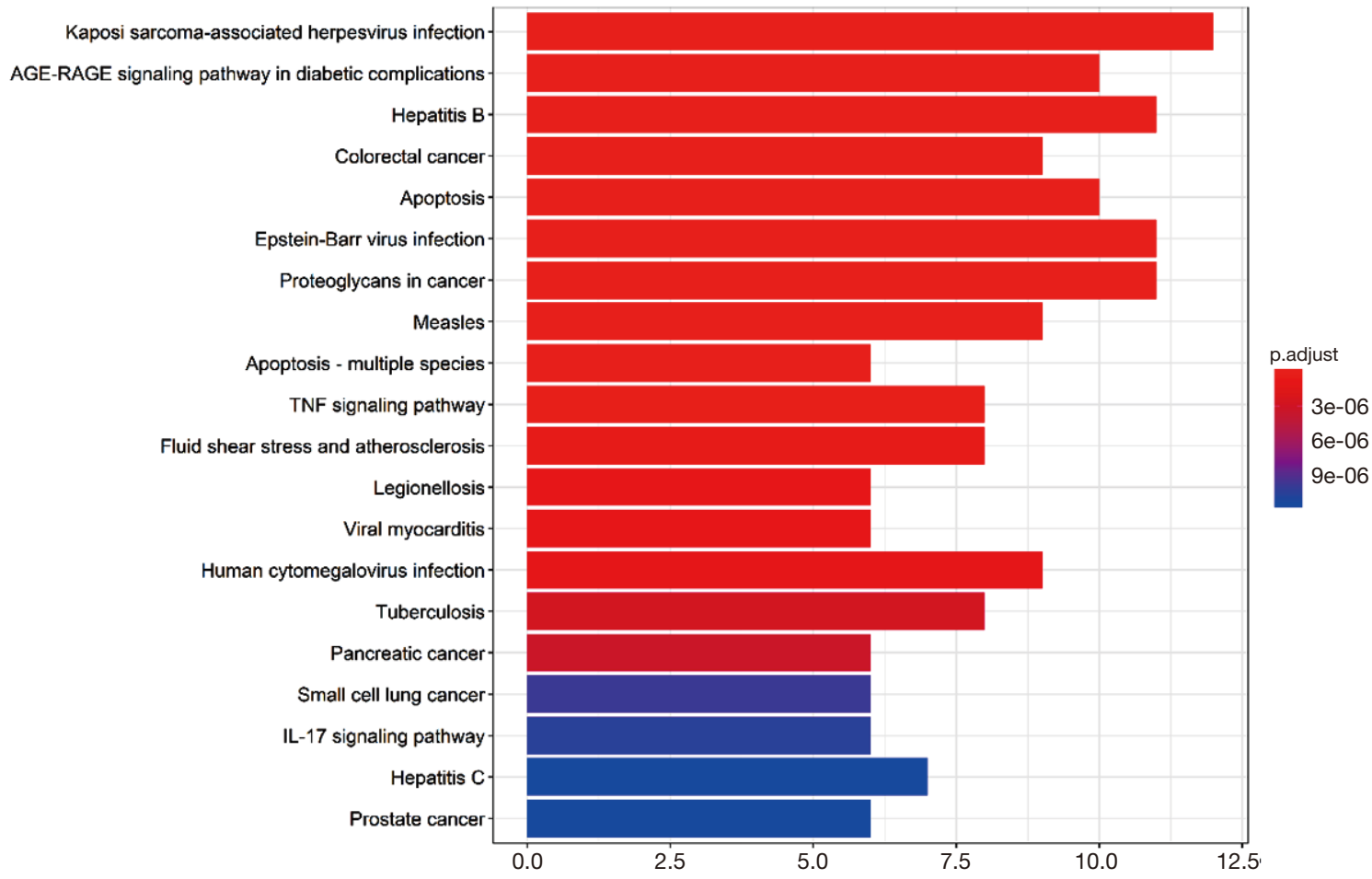

Figure 8 Histogram of KEGG pathway analysis.

these symptoms, while both the symptoms and the roots are simultaneously considered. Satisfactory effects have been obtained in the clinical application of the decoction intervention. The investigators' previous study revealed that BHD could reduce the expression of various proinflammatory factors and key protein molecules in the degenerative intervertebral disc of rats while simultaneously controlling the degeneration of the intervertebral disc.

In the present study, TCMSP, BATMAN-TCM, GeneCards, STRING, and other biological information databases were used preliminarily to explore the mechanism of BHD-regulating IDD. The study screened 131 interaction targets of the effective components of BHD, and 31 were targeted at degenerative disc disease. Of these 31 targets, IL6, VEGFA, and CASP 3 appeared most frequently. IL6 is confirmed to be involved in the degradation of the extracellular matrix in IDD (14). It is one of the most studied cytokines in the study of IDD. VEGFA may be co-expressed with the p 53 gene in degenerative disc tissue and co-acts in neovascularization and infiltration to accelerate the degeneration of intervertebral disc tissues (15). CASP3 is mainly involved in the initiation of apoptosis in the process of IDD (16).
From the interaction network diagram of the active component-intervertebral disc degeneration-target, 77 nodes and 384 edges were obtained. These reflected the complex network characteristics of multiple targets and multiple mechanisms of BHD in the intervention of IDD. The results of the GO enrichment analysis of the molecular function of the common targets of BHD and IDD also revealed that these top-ranked components were mainly molecules with the function of protein binding. The enrichment analysis of the KEGG biological pathway revealed that the signal pathways with the most target enrichment of BHD intervention on IDD were the AGERAGE signaling pathway, TNF signaling pathway, PI3K Akt signaling pathway, and MAPK signaling pathway. The TNF and MAPK signaling pathways often play an important role in the process of IDD by promoting the secretion of inflammatory factors, the apoptosis of pulpiform nucleus cells, and the degradation of the extracellular matrix through synergic effects (17-19). The PI3K Akt signaling pathway can delay the degeneration of the intervertebral disc by regulating the expression of MMP-3 (20) while inhibiting the apoptosis of pulpiform nucleus cells (21). The results from domestic and foreign 


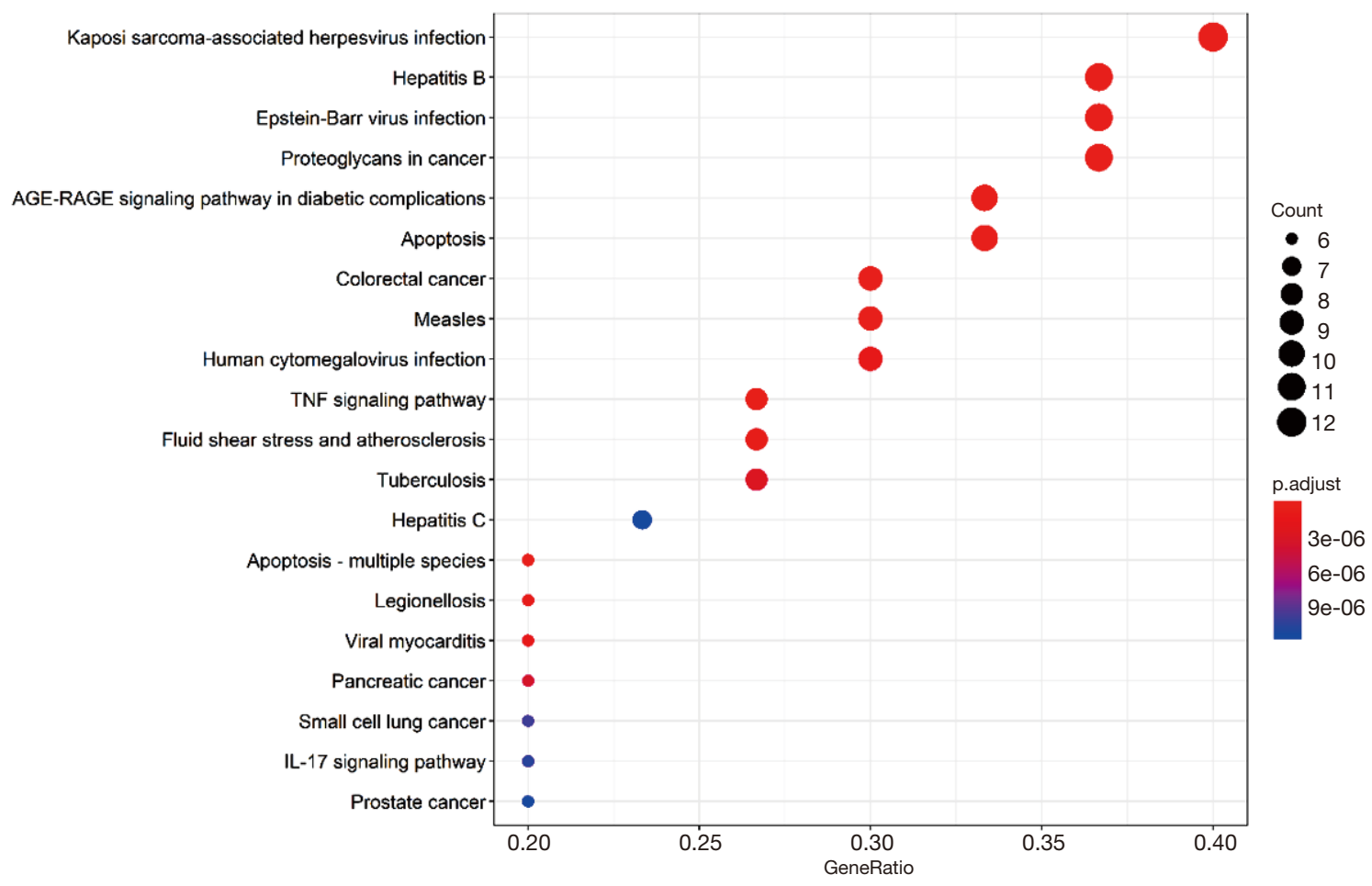

Figure 9 Bubble chart of KEGG pathway analysis.

literature revealed that there are fewer studies on the correlation between the AGE-RAGE signaling pathway and IDD. Xunlu et al. $(21,22)$ revealed, through integrating biological information, that the AGE-RAGE signaling pathway may play an important role in the process of IDD. This suggests the next direction of study on the mechanism of the development of IDD.

In summary, BHD intervenes in 31 targets in the process of IDD through 164 active ingredients and regulates various molecular functions and signal pathways through these targets while accordingly delaying the degeneration of the intervertebral disc. These findings provide a theoretical basis for further experimental studies on BHD in treating degenerative disc disease. The results of the KEGG enrichment analysis also revealed that the intervention targets of BHD on IDD frequently appeared in Kaposi's sarcoma-related herpes virus infection, hepatitis B, EpsteinBarr virus infection, and tumor proteoglycan. Whether the active components in BHD can regulate these pathological processes needs further study.

However, the regulatory targets and mechanisms of BHD on IDD summarized in this study are based on the current bioinformatics database, and the research on the pathogenesis of IDD lacks innovative findings. In addition, this study is based on the database information analysis and prediction of BHD intervention mechanisms related to IDD, which belongs to theoretical research. Further experiments are needed to verify these theoretical results.

\section{Conclusions}

This study screened the effective components, targets, and mechanisms of BHD in treating IDD using the network pharmacological technology while providing a theoretical basis for further experimental studies on BHD in treating degenerative disc disease.

\section{Acknowledgments}

Funding: This study was supported by Key Project of Hunan Traditional Chinese Medicine Research (No. 201902).

\section{Footnote}

Reporting Checklist: The authors have completed the MDAR reporting checklist. Available at http://dx.doi.org/10.21037/ 
apm-20-2586

Data Sharing Statement: Available at http://dx.doi. org/10.21037/apm-20-2586

Conflicts of Interest: All authors have completed the ICMJE uniform disclosure form (available at http://dx.doi. org/10.21037/apm-20-2586). The authors have no conflicts of interest to declare.

Ethical Statement: The authors are accountable for all aspects of the work in ensuring that questions related to the accuracy or integrity of any part of the work are appropriately investigated and resolved. The study was conducted in accordance with the Declaration of Helsinki (as revised in 2013). The study was approved by ethics board of Affiliated Hospital of Hunan Academy of Chinese Medicine (No. LLBH-201910310001) and informed consent was taken from all the patients.

Open Access Statement: This is an Open Access article distributed in accordance with the Creative Commons Attribution-NonCommercial-NoDerivs 4.0 International License (CC BY-NC-ND 4.0), which permits the noncommercial replication and distribution of the article with the strict proviso that no changes or edits are made and the original work is properly cited (including links to both the formal publication through the relevant DOI and the license). See: https://creativecommons.org/licenses/by-nc-nd/4.0/.

\section{References}

1. Sampara P, Banala RR, Vemuri SK, et al. Understanding the molecular biology of intervertebral disc degeneration and potential gene therapy strategies for regeneration: a review. Gene Ther 2018;25:67-82.

2. Ma X, Lin Y, Yang K, et al. Effect of lentivirus-mediated survivin transfection on the morphology and apoptosis of nucleus pulposus cells derived from degenerative human disc in vitro. Int J Mol Med 2015;36:186-94.

3. Zhu LG, Zhang P, Song QH, et al. Preliminary study of intervention in effect of Bushen Huoxue recipe on calcification of lumbar vertebra cartilage endplate of the aging gerbils. Zhongguo Gu Shang 2017;30:926-32.

4. Li NH, Xia L, Yi S, et al. Effect of Bushenhuoxue formula on interleukin-1 beta and discoidin domain receptor 2 levels in a rat model of osteoarthritis. J Tradit Chin Med 2015;35:192-6.
5. Ma DW, Xie XJ, Li XW, et al. Effects of bushenhuoxue on activities of glutamine synthetase and retinal Müller cells under TGF- $\beta \_2$-intervene hypoxic condition. Chin Ophthalmic Res 2010;28:407-11.

6. Wang PE, Zhang L, Ying J, et al. Bushenhuoxue formula attenuates cartilage degeneration in an osteoarthritic mouse model through TGF- $\beta / M M P 13$ signaling. J Transl Med 2018;16:72.

7. Zhao Q, Wan YG, Wang CJ, et al. Regulatory mechanism of p38MAPK signaling pathway on renal tissue inflammation in chronic kidney disease and interventional effect of traditional Chinese medicine. Zhongguo Zhong Yao Za Zhi 2012;37:1700-4.

8. Akeda K, Yamada J, Linn ET, et al. Platelet-rich plasma in the management of chronic low back pain: a critical review. J Pain Res 2019;12:753-67.

9. Vo NV, Hartman RA, Patil PR, et al. Molecular mechanisms of biological aging in intervertebral discs. J Orthop Res 2016;34:1289-306.

10. Millward-Sadler SJ, Costello PW, Freemont AJ, et al. Regulation of catabolic gene expression in normal and degenerate human intervertebral disc cells: implications for the pathogenesis of intervertebral disc degeneration. Arthritis Res Ther 2009;11:R65.

11. Takegami N, Akeda K, Yamada J, et al. RANK/RANKL/ OPG system in the intervertebral disc. Arthritis Res Ther 2017;19:121.

12. Hoyland JA, Le Maitre C, Freemont AJ. Investigation of the role of IL-1 and TNF in matrix degradation in the intervertebral disc. Rheumatology (Oxford) 2008;47:809-14.

13. Le Maitre CL, Freemont AJ, Hoyland JA. The role of interleukin-1 in the pathogenesis of human intervertebral disc degeneration. Arthritis Res Ther 2005;7:R732-45.

14. Ji ML, Lu J, Shi PL, et al. Dysregulated miR-98 Contributes to Extracellular Matrix Degradation by Targeting IL-6/STAT3 Signaling Pathway in Human Intervertebral Disc Degeneration. J Bone Miner Res 2016;31:900-9.

15. Lu XY, Ding XH, Zhong LJ, et al. Expression and significance of VEGF and p53 in degenerate intervertebral disc tissue. Asian Pac J Trop Med 2013;6:79-81.

16. Zhang K, Ding W, Sun W, et al. Beta1 integrin inhibits apoptosis induced by cyclic stretch in annulus fibrosus cells via ERK1/2 MAPK pathway. Apoptosis 2016;21:13-24.

17. Zhang J, Wang XL, Liu H, et al. TNF- $\alpha$ enhances apoptosis by promoting $\mathrm{CHOP}$ expression in nucleus pulposus cells: role of the MAPK and NF- $\kappa \mathrm{B}$ pathways. J Orthop Res 2019;37:697-705. 
18. Cao Z, Chen L. Inhibition of miR-27a suppresses the inflammatory response via the p38/MAPK pathway in intervertebral disc cells. Exp Ther Med 2017;14:4572-8.

19. Chen B, Wang HT, Yu B, et al. Carthamin yellow inhibits matrix degradation and inflammation induced by LPS in the intervertebral disc via suppression of MAPK pathway activation. Exp Ther Med 2017;14:1614-20.

20. Liu Z, Zhou K, Fu W, et al. Insulin-Like Growth Factor 1 Activates PI3k/Akt Signaling to Antagonize Lumbar Disc Degeneration. Cell Physiol Biochem 2015;37:225-32.

Cite this article as: Feng SH, Xie F, Yao HY, Wu GB, Sun XY, Yang J. The mechanism of Bushen Huoxue decoction in treating intervertebral disc degeneration based on network pharmacology. Ann Palliat Med 2021;10(4):3783-3792. doi: 10.21037/apm-20-2586
21. Xunlu Y, Minshan F, Liguo Z, et al. Integrative Bioinformatics Analysis Reveals Potential Gene Biomarkers and Analysis of Function in Human Degenerative Disc Annulus Fibrosus Cells. Biomed Res Int 2019;2019:9890279.

22. Jiang Y, Xie Z, Yu J, et al. Resveratrol inhibits IL$1 \beta$-mediated nucleus pulposus cell apoptosis through regulating the PI3K/Akt pathway. Biosci Rep 2019;39:BSR20190043. 